\title{
Diagnostic Levels in Dental Public Health Planning
}

\author{
Amid Ismail \\ School of Dentistry, University of Michigan, Ann Arbor, Mich., USA
}

\section{Key Words}

Dental caries, diagnosis · Public health · Epidemiology

of caries stages - Dental caries, prevention

\begin{abstract}
This concept paper discusses the rationale for using different diagnostic criteria for dental caries in public health programs. The author contends that epidemiological data or needs assessment surveys should collect data to provide information on the epidemiology of different stages of the caries process in order to enable planners to design tailored programs to prevent dental caries. In a rapidly progressing caries environment, conventional approaches to delivering preventive measures may not work. The author also contends that dental public health programs should expand their vision to influence how dentists are detecting, diagnosing and managing dental caries. Dentists' restorative decisions have significant impact on the oral health status of a nation. Henceforth, detecting the early or noncavitated caries level and preventing these lesions from progressing to the cavitated stage (or being restored) could have a significant impact on the oral health status around the world. There is a need for more research on the best methods to detect, prevent and treat early carious lesions.
\end{abstract}

Copyright $(2004$ S. Karger AG, Basel

\begin{tabular}{ll}
\hline KARGER & ( ) 2004 S. Karger AG, Basel \\
0008-6568/04/0383-0199\$21.00/0 \\
$\begin{array}{l}\text { Fax +4161306 12 34 } \\
\begin{array}{l}\text { E-Mail karger@karger.ch } \\
\text { www.karger.com }\end{array}\end{array}$ & $\begin{array}{l}\text { Accessible online at: } \\
\text { www.karger.com/cre }\end{array}$
\end{tabular}

Dental public health planning and practice around the world are based on different concepts, goals and approaches. All dental public health programs share common principles. The bedrock of successful planning in dental public health is information on the epidemiology of a disease and the modifiable risk factors that could be targeted to prevent it. The first step in planning of public health programs is to collect and analyze information on the prevalence or incidence and risk factors of a disease. Measurement of these key markers is an important first step in public health planning. Unfortunately, in most countries, the dental public health community has paid little or even no attention to the complex issues associated with measuring, detecting or diagnosing dental caries.

\section{What Is the Case Definition of Dental Caries?}

In spite of the progress in understanding the caries process, there is still some significant level of confusion among members of the dental community on what is dental caries. During most of the 20th century, dental caries was detected and managed as if the caries process is synonymous with 'cavities'. The practice of dentistry has focused on developing the best 'drill and fill' interventions. Even today some European and American re-

Prof. A. Ismail

School of Dentistry, University of Michigan

1011 N. University Ave., D2361

Ann Arbor, MI 48109-1078 (USA)

Tel. +1 734647 9190, Fax +1 734936 1597, E-Mail ismailai@umich.edu 
searchers and public health specialists still focus on the end stage of the caries process.

In my opinion, planning public health programs that focus only on the prevention of cavitated carious lesions could, sometimes, be detrimental to oral health. In this paper, and for reasons to be presented in the next sections, I propose that we should consider and define the stages of dental caries in prevention, health promotion or treatment programs. A stage-based prevention and management of dental caries represents a new approach in planning dental public health programs in the 21 st century.

Failure to consider the stages of the caries process when designing prevention and treatment programs could lead to inappropriately removing healthy tooth structure in a tooth with a carious lesion that could remineralize. There is evidence that dentists have different levels of knowledge on caries etiology and prevention as well as on when to restore a tooth [Moon et al., 1998; Lewis et al., 1997; Mejàre et al., 1999]. Though these findings have been known since the early 1980s [Elderton, 1985], they have not yet been the subject of debate among dental public health experts. The misclassification of sound tooth surfaces due to relatively less than perfect specificity of detection tools and criteria of dental caries has a significant impact on public health. Even a small percentage of false-positive classifications of teeth can result in a significant contribution to the restorative burden in individuals who predominately have sound teeth. Filling sound teeth, due to misdetection or misclassification, leads to a spurious increase in dental caries indices [Ismail et al., 1997]. This misclassification problem should present an ethical dilemma for promoters of health.

In a longitudinal study of restorative decisions made by a sample of private practitioners in Montreal, Canada, it was found that $50 \%$ of new class I restorations placed in maxillary first permanent molars of 6- to 9-year-old children within a period of 3 months after the baseline examination were placed in clinically sound teeth (with no signs of staining, active or arrested early noncavitated or cavitated carious lesions) [Ismail et al., 1997]. For mandibular permanent molars, $33 \%$ of class I restorations were placed in sound teeth within 3 months after the baseline examination. In the first 3 months of the second year of followup, it was found that the majority of class I restorations were placed in noncavitated pits and fissures $(68.8 \%$ for maxillary molars and $31.8 \%$ for mandibular molars). In this study, the trained and calibrated dental examiners were instructed to score high when in doubt to give the benefit of any doubt in classification of the caries status of a tooth surface to the dentists who provided the dental care for each child enrolled in the study. If this degree of error due to misclassification of caries detection in clinical practice is found in other countries, then we have an ethical problem that we so far have refrained from discussing.

In my opinion, it is unfortunate that the current standard for detection and assessment of dental caries in planning for dental public health programs in most countries is based on the WHO [1997] or NIDCR [Radike, 1968; NIDCR, 1987] criteria which measure dental caries at the cavitation or 'softness' level. As a result, all countries have data on the prevalence of cavitated carious lesions but not the prevalence of noncavitated or early carious lesions. In my opinion, having the latter information may help dental public health programs to design targeted secondary prevention programs to prevent the progression of carious lesions. Such programs are particularly important to promote the dental health of young children and patients who experience rapid progression of dental caries. The issue of what detection level should be used in dental public health planning has not been widely discussed maybe because it has been assumed that 'caries is caries' and public health programs should be focused on 'frank' cavitated lesions. Since the goal is to prevent 'cavities', assessment should focus on measuring their presence. I contend that focusing only on cavitation misses the most important stage of the caries process: early enamel carious lesions.

\section{What Is a Public Health Problem?}

In deciding what detection levels of dental caries should be used in planning dental public health programs, it is important to define what a public health problem is. Public health, as Burt and Eklund [1999] contend, cannot easily be defined. There is a general consensus that public health is '...fulfilling society's interest in assuring conditions in which people can be healthy' [Institute of Medicine, 1988]. This definition implies that public health planning should encompass all programs or services that can promote the health status of a targeted population. Public health programs or services should include activities that focus on health promotion and primary, secondary and tertiary health care.

Defining a public health problem is also difficult because there are various cultural, political, personal, community and organizational factors that should be considered for labeling any health condition as a "public health problem'. Burt and Eklund [1999] propose that a 
public health problem should meet the following two conditions:

(1) there is a condition or situation that is widespread and has an actual or potential cause of morbidity or mortality;

(2) there is a perception on the part of the public, government or public health authorities that the condition is a public health problem.

Dental caries is a public health problem because it is a widespread condition that is costly to treat and it impacts on the quality of life at all ages. In most of the developing world, dental caries remains untreated [Carino et al., 2003; Yee and Sheiham, 2002]. Fortunately, unlike other infectious diseases, dental caries does not cause significant public alarm because of its low mortality and impact on daily living. Nevertheless, the social and economic costs of dental caries are significant [Yee and Sheiham, 2002].

It is time to rethink the scope of dental public health planning. Traditionally, public health programs have been organized to prevent deadly infectious diseases or promote health by programs designed to reduce the exposure to risk factors in populations. Assessment, policy development and assurance are the three major functions of public health agencies [Institute of Medicine, 1988]. This basic view of public health should be modified to incorporate all activities that the public or health professionals undertake to promote health, prevent disease or manage the damage caused by a disease. Dental public health programs should encompass a wide range of activities throughout life. Dental public health plans should address the following questions:

(1) What programs are needed to promote oral health and healthy behaviors?

(2) What programs are needed to prevent the development of early carious lesions in enamel?

(3) What programs are needed to prevent the progression or reverse early carious lesions in enamel?

(4) What programs are needed to minimally treat cavitation limited to enamel?

(5) What programs are needed to minimally treat or prevent the progression of noncavitated lesions in enamel and in dentin?

(6) What programs are needed to minimally treat cavitated carious lesions?

And most importantly:

(7) What tools should be used to eliminate the misclassification of dental caries and reduce errors in decision making?

Diagnostic Levels in Public Health

Planning
These questions present a model for dental public health planning that is different from the current understanding of the scope of dental public health in some countries. However, we are in the 21 st century and on the verge of a significant revolution in diagnostics, regeneration of tissues and genetic testing and therapy. We need to change the current paradigm used in dental public health programs. Such programs should be tailored to the disease status of a targeted population. When the prevalence of noncavitated carious lesions is high, more rapid and progressive prevention programs should be designed than when the prevalence of noncavitated lesions is low. Of course, the prevalence of noncavitated in relation to cavitated carious lesions should be taken into consideration. In developed countries, such as Canada [Ismail et al., 1992], epidemiological data indicate that the ratio of noncavitated to cavitated lesions is higher than in economically developing countries such as Lithuania [Machiulskiene et al., 1998]. Hence in the former, the emphasis should be on conservative methods of management as well as on tailored individualized prevention. In countries where the rate of progression from the noncavitated stage to cavitation is high, the emphasis should also be on tailored but more extensive prevention as well as general preventive measures (such as water or salt fluoridation). While I admit that these decisions are made based upon my expert opinion, I strongly advocate that we start to consider tailoring of dental public health programs based upon disease level and distribution of the different stages of dental caries.

\section{Detection Levels in Dental Public Health Programs}

The mission of dental public health planning should be to promote health, prevent the development of disease, prevent the progression of early disease, treat the sequelae of a disease and restore functional health. Dental caries is a process, and its assessment should be determined based on population characteristics and the burden of disease in areas where a public health program is to be implemented. The stages of dental caries that should be considered in public health programs should include enamel and dentinal carious lesions at the noncavitated and cavitated levels. It would also be important in the future to include measures of caries activity. A decision on what stage to target and measure should be made for each program based upon the goals and logistics of the area where the program is to be implemented. Enamel carious lesions can 
be included in detection protocols used in programs targeting early childhood caries and primary prevention programs; dentinal noncavitated lesions can be the focus of public health programs that aim to reduce the surgical management of dental caries, and cavitated lesions can be the focus of programs that aim to prevent the end stage of the caries process. While the prevention of carious cavitation is the ultimate goal of all public health programs, using earlier stages of the caries process may help shift the focus from universal prevention towards targeted prevention and from the 'drill and fill' approach to the 'conservative' approach of managing dental caries.

The focus on high-caries risk populations in public health programs will not result in significant improvement in dental health until we understand that preventive programs that focus only on preventing carious cavitation cannot always succeed in changing the burden of dental caries. The failure to consider the stage of progression of dental caries may be one of the reasons why preventive programs targeting high-caries individuals have not been successful (an example of such programs can be found in Watson et al. [1999]).

\section{Moving Forward}

It is sometimes tempting to suggest new models and how to do things. The challenge is how to change and sustain the change. The dental community, especially its academic corps, is slow to change and is extremely conservative. While frequent experimentation with patients using new gadgets and untested concepts is common, dental professionals have been slow to adopt new scientific findings, especially those that are not related to the surgical model of care or do not fit within the context of a dental practice environment. While it is beyond the scope of this paper to analyze the causes and solutions to the current dilemmas facing dental education, dental public health, dental practice and dental research, it is important that we take actions to improve the status quo.

As discussed in this paper, there is a need for research and programs to promote the early detection of dental caries in order to prevent its rapid progression in some populations, to decrease the false-positive detection rates associated with current caries detection systems, to promote nonsurgical approaches for the management of early carious lesions, to use microsurgical approaches to treat small cavitated lesions and to increase access to primary, secondary and tertiary care.

Dental public health should shift its sole focus on highrisk groups and populations to all risk groups and all individuals within populations. I am not at all advocating that dental public health programs should change their emphasis on preventing 'cavities'; rather, I suggest that for some population groups, such as young children and medically compromised patients, there is a need to focus as well on preventing early carious lesions. While there is scarcity of evidence on how to prevent these lesions from progressing to cavitation, secondary preventive programs may be designed with a different frequency and mode of delivery than the design of a similar program targeting only cavitated carious lesions. In the face of a rapidly progressing disease scenario, dental professionals should not rely on 'simple preventive measures' [Machiulskiene et al., 1998]. Rather there would be a need to augment a preventive program with dietary counseling, use of alternative sweeteners, frequent professional tooth cleaning and even the use of antimicrobials. Such measures may not be needed if the overall caries activity is low.

Finally, while enamel caries was recognized by G.V. Black in 1909 [Black, 1910] as the most important stage in the caries process, the dental public health community, dental educators, researchers and practitioners have not paid much attention to how to detect and manage these lesions. This area should receive significantly more research emphasis.

\section{References}

Black G: A plea for greater earnestness in the study of caries of the enamel in its relation to the practice of dentistry. Dent Brief 1910;15:161178.

Burt BA, Eklund SA: Dentistry, Dental Practice, and the Community. Philadelphia, Saunders, 1999, pp 34-36.
Carino KMG, Shinada K, Kawaguchi Y: Early childhood caries in northern Philippines. Community Dent Oral Epidemiol 2003;31:81-89.

Elderton RJ: Implications of recent dental health services research on the future of operative dentistry. J Public Health Dent 1985;45:101105.

Institute of Medicine: The Future of Public Health. Washington, National Academy Press, 1988.
Ismail AI, Brodeur JM, Gagnon P, Payette M, Picard D, Hamalian T, Olivier M, Eastwood BJ: Prevalence of non-cavitated and cavitated carious lesions in a random sample of 7-9-yearold schoolchildren in Montreal, Quebec. Community Dent Oral Epidemiol 1992;20:250 255. 
Ismail AI, Brodeur JM, Gagnon P, Payette M, Picard D, Hamalian T, Olivier M: Restorative treatments received by children covered by a universal, publicly financed, dental insurance plan. J Public Health Dent 1997;57:11-18.

Lewis DW, Pharoah MJ, El-Mowafy O, Ross DG: Restorative certainty and varying perceptions of dental caries depth among dentists. J Public Health Dent 1997;57:243-245.

Machiulskiene V, Nyvad B, Baelum V: Prevalence and severity of dental caries in 12-year-old children in Kaunas, Lithuania 1995. Caries Res 1998;32:175-180.
Mejàre I, Sundberg H, Espelid I, Tveit AB: Caries assessment and restorative treatment thresholds reported by Swedish dentists. Acta Odontol Scand 1999;57:149-154.

Moon H, Paik D, Horowitz AM, Kim J: National survey of Korean dentists' knowledge and opinions: Dental caries etiology and prevention. J Public Health Dent 1998;58:51-56.

National Institute for Dental and Craniofacial Research (NIDCR): Oral Health of United States Adults. Bethesda, NIDCR, 1987, pp 161-165.

Radike AW: Criteria for diagnosing dental caries; in American Dental Association (ed): Proceedings of the Conference on the Clinical Testing of Cariostatic Agents. Chicago, American Dental Association, 1968, pp 87-88.
Watson MR, Howoritz AM, Garcia I, Canto MT: Caries conditions among 2-5-year-old immigrant Latino children related to parents' oral health knowledge, opinions and practices. Community Dent Oral Epidemiol 1999;27:815.

World Health Organization: Oral Health Surveys: Basic Methods. Geneva, WHO, 1997, pp 4142.

Yee R, Sheiham A: The burden of restorative dental treatment for children in third world countries. Int Dent J 2002;52:1-9. 